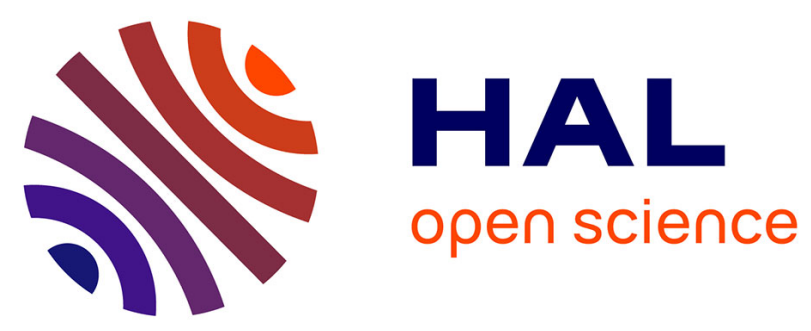

\title{
Confrontation between Molecular Dynamics and micromechanical approaches to investigate particle size effects on the mechanical behaviour of polymer nanocomposites
}

Vincent Marcadon, David Brown, Éveline Herve, Patrice Mele, Nicole

Dominique Albérola, André Zaoui

\section{To cite this version:}

Vincent Marcadon, David Brown, Éveline Herve , Patrice Mele, Nicole Dominique Albérola, et al.. Confrontation between Molecular Dynamics and micromechanical approaches to investigate particle size effects on the mechanical behaviour of polymer nanocomposites. Computational Materials Science, 2013, 79, pp.495-505. 10.1016/j.commatsci.2013.07.002 . hal-02350682

\section{HAL Id: hal-02350682 https://hal.science/hal-02350682}

Submitted on 13 Jan 2020

HAL is a multi-disciplinary open access archive for the deposit and dissemination of scientific research documents, whether they are published or not. The documents may come from teaching and research institutions in France or abroad, or from public or private research centers.
L'archive ouverte pluridisciplinaire HAL, est destinée au dépôt et à la diffusion de documents scientifiques de niveau recherche, publiés ou non, émanant des établissements d'enseignement et de recherche français ou étrangers, des laboratoires publics ou privés. 


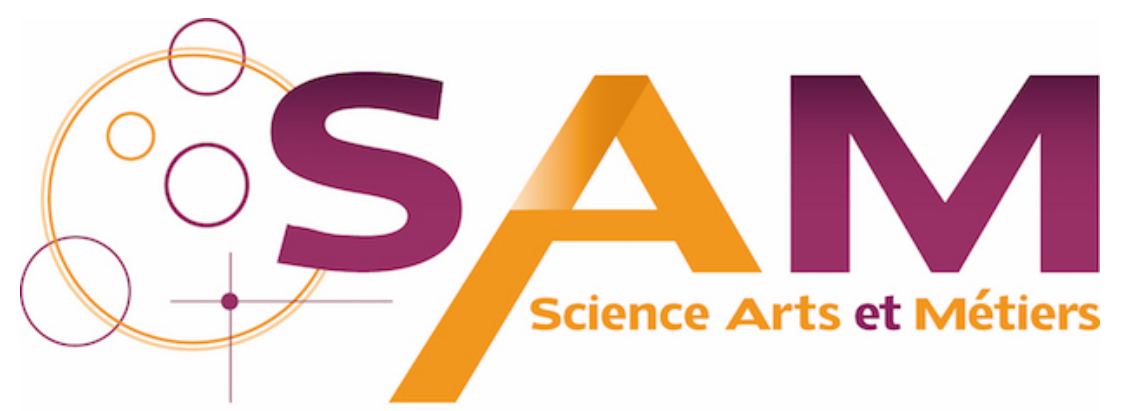

Archive Ouverte - Open Repository

\section{Science Arts \& Métiers (SAM)}

is an open access repository that collects the work of Arts et Métiers ParisTech researchers and makes it freely available over the web where possible.

This is an author-deposited version published in: https://sam.ensam.eu Handle ID: .http://hdl.handle.net/null

\section{To cite this version :}

Vincent MARCADON, David BROWN, Éveline HERVE , Nicole Dominique ALBÉROLA, André ZAOUI - Confrontation between Molecular Dynamics and micromechanical approaches to investigate particle size effects on the mechanical behaviour of polymer nanocomposites Computational Materials Science - Vol. Volume 79, p.Pages 495-505 - 2013 


\title{
Confrontation between Molecular Dynamics and micromechanical approaches to investigate particle size effects on the mechanical behaviour of polymer nanocomposites
}

\author{
V. Marcadon ${ }^{a, *}$, D. Brown ${ }^{b}$, E. Hervée $^{\mathrm{a}, \mathrm{c}, \mathrm{d}}$, P. Mélé ${ }^{\mathrm{b}}$, N.D. Albérola ${ }^{\mathrm{b}}$, A. Zaoui ${ }^{\mathrm{a}, \mathrm{e}}$ \\ ${ }^{a}$ Laboratoire de Mécanique des Solides, Ecole Polytechnique, CNRS UMR 7649, F-91128 Palaiseau Cedex, France

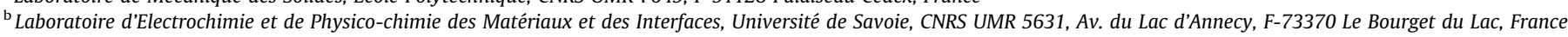 \\ ${ }^{\mathrm{C}}$ Université de Versailles Saint-Quentin en Yvelines, 45 Avenue des Etats-Unis, F-78035 Versailles Cedex, France \\ ${ }^{\mathrm{d}}$ Centre des Matériaux, Mines ParisTech, CNRS UMR 7633, BP 87, F-91003 Evry Cedex, France \\ e Laboratoire Procédés et Ingénierie en Mécanique et Matériaux, Arts et Métiers ParisTech, CNRS UMR 8006, 151 Boulevard de l'Hôpital, F-75013 Paris, France
}

Keywords:

Particulate nanocomposites

Size effects

Mechanical behaviour

Micromechanical models

Molecular Dynamics

\begin{abstract}
A B S T R A C T
This paper aims at developing a method to account for a particle size effect on the mechanical behaviour of particulate nanocomposites. An introduction of specific information at the atomic scale, through Molecular Dynamics (MD) simulations, into homogenisation models of the overall mechanical behaviour of heterogeneous materials (micromechanical approaches) is proposed here. By studying virtual nanocomposites, which consist of silica inclusions embedded in a polymer matrix, MD simulations have shown the existence of an interphase of disturbed matrix surrounding the inclusions, whose thickness depends neither on the inclusion size nor on the volume fraction of inclusions. By considering this interphase of fixed thickness, a particle size effect is then deduced from a dilute micromechanical model which derives from the classical Eshelby's inhomogeneity problem. Effective elastic moduli of the composite strongly vary with the particle size for a fixed volume fraction of particles. Nevertheless, opposite trends are observed relative to the interphase behaviour. Whereas effective moduli increase with the particle size for an interphase softer than the matrix, they decrease in the reverse case. The confrontation between MD and micromechanical approaches and the characterisation of the interphase elastic moduli by an inverse method allow the stiffening effects observed by MD to be explained in the case of an interphase softer than the pure matrix.
\end{abstract}

\section{Introduction}

In materials science, size effect (grain size in metallic materials or filler size in composites for instance) is an important issue to improve mechanical properties of heterogeneous materials. This field of research has already been investigated for many years. Nevertheless, with the development of nanomaterials (materials where characteristic lengths vary from a few nanometres to a few hundred nanometres), scientists and industrials are confronted with smaller scales, hence with new physical and mechanical phenomena. Such nanomaterials are expected to exhibit specific mechanical properties and their development for various applications gives rise to a lot of interest. In the present work emphasis is on nanocomposites with polymer matrices.

* Corresponding author. Present address: Onera - The French Aerospace Lab, DMSM, 29 Av. de la Division Leclerc, BP 72, F-92322 Châtillon Cedex, France. Tel.: +33 146734524; fax: +33 146734891.

E-mail address: Vincent.Marcadon@onera.fr (V. Marcadon).
Whereas elaboration processes to add filler particles of micronic size to various polymer matrices are well controlled, it is no longer the case when adding nanoscopic fillers. At this smaller scale, interactions between particles and polymer chains or between particles themselves play an important role. Thus new phenomena induced by physical interactions appear and govern the elaboration process. According to review articles, see Móczó and Pukánsky [1] or Fu et al. [2] for instance, the latter focusing on particulate polymer composites, a strong coupling between different phenomena exists. Effects of the size, the volume fraction and the aggregation of the particles are often combined and depend considerably on physico-chemical interactions between the particles and the matrix, the processing route of the composite, and the presence or not of coupling agents. Interfacial interactions between fillers and matrices seem to be critical parameters governing the effective behaviour of nanocomposites [3].

Chen et al. [4], studying epoxy resins reinforced with silica nanoparticles, have shown that, whereas effective Young's modulus of the nanocomposites raises with the filler content for small 
nanoparticles of $6 \mathrm{~nm}$ radius, it decreases when increasing the filler content in the case of large nanoparticles of $50 \mathrm{~nm}$ radius, due to stress concentration and plasticity in the matrix surrounding the particles. Cho et al. [5] asserted the specificity of the nanoscale studying nano and microcomposites consisting of a vinyl ester resin filled with either glass beads or spherical alumina particles. An increase of effective Young's modulus was observed when decreasing the particle size in the case of the nanocomposites. On the contrary, no similar effect was observed in the case of the classical microcomposites. They have also shown an improvement in the mechanical properties of composites with the volume fraction of fillers. Nevertheless, despite some recent progress in the processing of perfectly dispersed nanocomposites, see the work of Kongsinlark et al. [6] on monodispersed silica-polyisoprene nanocomposites for instance, most of the time a particle radius decrease entails an increase of the aggregation phenomenon. The relevance of coupled phenomena at the nanoscale (size effect and aggregation) has also been observed by Steenbrink et al. [7] studying styrene-acrylonitrile filled with soft polymeric core-shell inclusions. Different particle radii were considered varying from $100 \mathrm{~nm}$ to $600 \mathrm{~nm}$. In the case of the smallest particles, less than $150 \mathrm{~nm}$ radius, aggregation problems have been observed in contrast to the larger particle radii. Another specific process problem at the nanoscale is the surface treatment of the filler with grafting and coating agents; any change in the filler surface reactivity implies a modification of the chain mobility near the filler, see the works of Albérola et al. [8] and Lazzeri et al. [9] for instance.

One specificity of nanocomposites, in comparison to classical ones (i.e., with micronic size particles), is the fact that an interphase of disturbed matrix, because of coupling agents or due to the presence of the fillers, exists surrounding the fillers and its range is no longer negligible at this scale. This interphase usually has specific chemical composition, physical and mechanical properties [1,2]. Berriot et al. [10,11], performing nuclear magnetic resonance measurements on rubbers filled with silica nanoparticles of $30 \mathrm{~nm}$ radius, have highlighted this interphase of disturbed matrix. Disturbances were observed on the local mobility of the polymer chains that substantially falls near the filler. The behaviour of this interphase depended on both the particle dispersion and the nature of the coupling agent. Such an interphase has also been predicted using Molecular Dynamics (MD) simulations, see the work of Starr et al. [12-14] for instance. A detailed study of the local disturbances induced by the presence of the nanoparticles has been performed by Barbier et al. [15] on poly(ethylene oxide) reinforced by silica nanoparticles. In contrast to previous molecular modelling studies, the silica model developed was an atomistic one. The analysis of the chain mobility using auto-correlation functions highlighted a diminution of the mobility in presence of a nanoparticle, the chain mobility vanishing drastically near the nanoparticle surface. Moreover, disturbances of the polymer matrix in the neighbourhood of the inclusion tended to a preferential reorientation of the polymer chains tangentially to the particle surface. By studying a large nanocomposite system made of a silica nanoparticle of radius $\approx 2.2 \mathrm{~nm}$ embedded in a 30,000 -site united-atom $\mathrm{CH}_{2}$ matrix, Brown et al. [16] observed for the bulk modulus of the system that adding rigid nanoparticles does not lead necessarily to an increase of the overall modulus of the nanocomposite in comparison to that of the pure matrix. Similar results have been obtained by Odegard et al. [17] through a confrontation between MD simulations and continuum mechanics. They also observed an interphase of disturbed matrix, whose range depended on the interaction between the silica particle and the matrix. The study of both Young's and shear moduli through MD revealed that, for any case of interaction between the particle and the matrix considered, the effective moduli of the composites were lower than the ones of the pure matrix despite the addition of rigid inclusions.
More recently, Ghanbari et al. [18] and Ndoro et al. [19], studying polystyrene composites filled with silica nanoparticles by MD, addressed the influence of the silica nanoparticles grafting on the interphase structure. On similar systems and by simulating tensile tests, Rahimi et al. [20] observed a stiffening effect of the silica nanoparticles increasing with their volume fraction, but also a reorientation of the polymer chains in the loading direction. Adnan et al. [21], modelling a polyethylene matrix filled with fullerene bucky-balls, have shown that nanocomposite effective Young's and bulk moduli increased when decreasing the particle size. A complementary study of Cho and Sun [22] has highlighted that such a size effect depends on the interaction between the inclusion and the matrix by varying the parameters of the modelling; the stronger the interaction, the more significant the size effect. Particle size effect in nanocomposites has been addressed through micromechanical approaches too. By considering the interphase of disturbed matrix mentioned in the literature, Marcadon et al. [23] have shown that a particle size effect in particulate nanocomposites can be modelled by using a morphologically representative pattern-based approach within classical continuum micromechanics. Boutaleb et al. [24,25] have proposed recently an original approach to discuss the issue of stress concentration and plasticity in the matrix surrounding the particles. They considered an interphase, having a given thickness, with a Young's modulus varying from the one of the particle to the one of the undisturbed matrix, and they have studied the particle size effect on nanocomposites effective Young's modulus and yield stress. By using approaches involving both the finite-element method and micromechanical models, Saber-Samandari and Afaghi-Khatibi [26] and, more recently, Peng et al. [27] have studied the influence of the volume fraction and the shape of the fillers on the elastic behaviour of particulate nanocomposites. In both cases, they assumed the existence of an interphase with properties grading from those of the filler to those of the matrix.

The purpose of the present work is to study the influence of particle size on the overall mechanical behaviour of filled polymers at the nanoscale. To avoid the practical difficulties involved in obtaining perfectly dispersed nanoparticles [5-7], and in order to characterise specifically a particle size effect (i.e., without any combined effects due to percolation for example), a molecular modelling approach is used in order to produce perfectly dispersed but virtual nanocomposites. After this first introduction section, the second part of this paper describes how the virtual nanocomposites have been prepared. The interphase of disturbed matrix surrounding these nanoparticles has already been studied [28] and its range provides a characteristic length of the nanoscale, the interphase thickness that equals about $2 \mathrm{~nm}$. Effective mechanical properties of the various nanocomposites generated are determined simulating mechanical tests by MD. The third part illustrates how the characteristic lengths of the studied nanocomposites (the interphase thickness and the particle radius) can be accounted for in a micromechanical approach and their effects on the overall behaviour of the nanocomposites are investigated. From a general point of view, adding rigid particles to a polymer matrix induces a stiffening effect. Nevertheless, depending on the behaviour of the interphase of disturbed matrix, it seems that either the stiffening effect can be emphasised when decreasing the particle size or can be softened, resulting even in an overall softening effect for some limit cases. To distinguish both these opposite trends, we use the terms over-stiffening and under-stiffening in the following. The fourth part deals with the confrontation between molecular modelling and micromechanical approaches to characterise the interphase behaviour and to explain the observed under-stiffening particle size effect. By fitting mechanical data supplied by MD thanks to the micromechanical model described in this paper, elastic moduli of the interphase are estimated. Particle size effect 
predicted by MD is discussed. It can be explained in terms of an interphase of disturbed matrix softer than the pure matrix.

\section{Modelling of virtual nanocomposites}

The following description of the method used to create model nanocomposites gives just the most relevant details of the molecular simulations for the purpose of this study. Extensive details of the molecular modelling aspects of the study of nanocomposites have already been published by Brown et al. [28].

\subsection{Silica nanoparticles}

The specific nature of the components of the composite is not important for this kind of generic study. We simply require a hard isotropic elastic particle embedded in an isotropic softer medium. For this reason a sample configuration of bulk amorphous $\mathrm{SiO}_{2}$ was first generated using standard two-body potentials $[29,30]$. We then replaced the two-body potentials with an explicit network of $\mathrm{Si}-\mathrm{O}$ bonds. By adjusting the strength of the $\mathrm{Si}-\mathrm{O}$ bonds and the stiffness of the $\mathrm{O}-\mathrm{Si}-\mathrm{O}$ and $\mathrm{Si}-\mathrm{O}-\mathrm{Si}$ valence angle potentials it was found possible to maintain a rigid structure with approximately the same radial distribution functions and, importantly, having about the same elastic moduli of the bulk 'atomic' model. The substitution of the two-body potentials by a small number of near-neighbour bonds and angles reduces enormously the computing time required and thus renders systems with larger particles much more accessible. Maintaining an atomic structure for the particle is also advantageous as it allows the elastic moduli of pure 'silica' to be determined. This is particularly important here where the elastic moduli of the pure silica and pure polymer phases are used as input into the continuum mechanics calculations. Pure silica simulations were performed using the same 30,375-atom system as constructed in our previous work [28].

This previous article explains in detail how nanoparticles with nominal radii of $R_{\mathrm{inc}}=1.5 \mathrm{~nm}, R_{\mathrm{inc}}=3 \mathrm{~nm}$ and $R_{\mathrm{inc}}=6 \mathrm{~nm}$ were subsequently created. As we maintain an atomic representation of the nanoparticles there is inevitably a certain amount of surface roughness. The subtle consequences of this on the volume fractions of filler have been discussed previously [28] and are important for this study.

\subsection{Polymer matrices}

The generic model of a polymer chain is one that has been often employed to successfully study amorphous polymer systems [31-34]. In this model, linear chains of $n$ united-atom $\mathrm{CH}_{2}$ sites are held together by rigid bonds, and valence angle and torsion angle potentials give the internal structure and rotational barriers. Non-bonded interactions are represented by a purely repulsive Weeks-Chandler-Andersen potential which operates for all intermolecular pair interactions and between all pairs of sites in the same chain separated by at least three intervening ones. As explained previously [28], to compensate for the lack of an attractive part to the potential, a hydrostatic pressure of 5000 bars is applied. This gives reasonable polymer densities and thus serves as a reference pressure for the mechanical tests. Full details of the preparation of the three pure matrix systems containing 10, 80 and 640 chains of $n=1000 \mathrm{CH}_{2}$ sites per chain have been given previously [28].

\subsection{Nanocomposites}

The method used to introduce a silica particle into a pre-relaxed polymer matrix has been described before in detail $[16,28]$. Briefly, a box of polymer is first expanded by a volume corresponding roughly to that of the particle that is to be inserted. A spherical cavity is then generated in the polymer matrix by gradually introducing a repulsive potential at the centre of the box. This creates a spherical space into which the silica particle can be inserted. The repulsive potential is then switched-off and the system allowed to relax.

By using such a technique, it was explained in our previous article [28] how three composite systems were prepared at a fairly low volume fraction of silica of about $4.5 \%$ by combining the 10 -chain polymer matrix with the $R_{\mathrm{inc}}=1.5 \mathrm{~nm}$ particle, the 80 -chain system with the $R_{\mathrm{inc}}=3 \mathrm{~nm}$ particle, and the 640 -chain system with the $R_{\text {inc }}=6 \mathrm{~nm}$ particle; these systems are used again here and are referred to as R15L, R30L and R60L, respectively. Two other systems at a much higher target volume fraction of $\approx 27 \%$ silica were also prepared combining the $R_{\text {inc }}=3 \mathrm{~nm}$ particle with the 10 -chain polymer matrix $(\mathrm{R} 30 \mathrm{H})$ and the $R_{\mathrm{inc}}=6 \mathrm{~nm}$ particle with the 80 -chain matrix (R60H). See Fig. 1(a) as an illustration of the nanocomposite systems.

\subsection{Simulation of mechanical tests}

All MD simulations were carried out using the gmq suite of codes (http://www.lmops.univ-savoie.fr/brown/gmq.html) with an integration time step of $2 \mathrm{fs}$. Bond length constraints were satisfied to a relative tolerance of $10^{-6}$. All simulations here were carried out under conditions of controlled pressure tensor $\underline{P}$ and temperature $T$. The temperature was maintained at $100 \mathrm{~K}$ using a standard loose-coupling algorithm with a time constant $\tau_{T}=1 \mathrm{ps}$ [35]. The systems were maintained close to the required pressure tensor using a loose-coupling technique in which the rate of change of the matrix $\underline{h}$, made up of the column vectors defining the shape and size of the MD box, are coupled to the difference between the measured and required pressure tensors:

$\underline{\underline{h}}(t)=\frac{\underline{\underline{P}}(t)-\underline{\underline{\underline{P_{\text {req }}}}}(t)}{\lambda \tau_{P}}$

where $\lambda$ is a pre-defined constant and $\tau_{P}$ is a relaxation time determining the rate at which the $\underline{h}$ matrix responds to the imbalance between the internally measured pressure tensor, $\underline{P}$, and that required, $\underline{P_{\text {req }}}$.

To determine the mechanical properties of the pure silica, the pure polymer matrices and the five composite systems, nonequilibrium experiments were performed by linearly changing components of the required pressure tensor in order to obtain bulk, shear and Young's moduli, and Poisson's ratio too. Despite some care must be taken when going from MD to continuum mechanics, because of strongly different timescales and the discrete representation of the matter through MD, the variations of the components of the pressure tensor and the $\underline{h}$ matrix, $-\left(P_{i j}(t)-P_{i j}\left(t_{0}\right)\right)$ and $\left(h_{i j}(t)-h_{i j}\left(t_{0}\right)\right) / h_{i j}\left(t_{0}\right)$, can be related to those of the classical macroscopic stress and strain tensors, $\Sigma_{i j}$ and $E_{i j}$ respectively, $t_{0}$ denoting the initial time of the non-equilibrium experiments. The quantities at $t_{0}$ are actually mean quantities computed on the baseline simulated just before the non-equilibrium experiments, see details below.

The different systems were assumed to be isotropic according to mechanical responses (see Fig. 1(b)) to allow the definition of the aforementioned elastic moduli. This assumption is relevant here and is discussed in Section 4.1. The rate of change of a required pressure tensor component was \pm 1 bar $\mathrm{ps}^{-1}$ in all cases. To ensure a good agreement between the measured and required pressure tensors, the values of $\tau_{P}$ were optimised for each system. It should be noted that Eq. (1) is formulated in an absolute way which means that equal pressure differences lead to relatively 


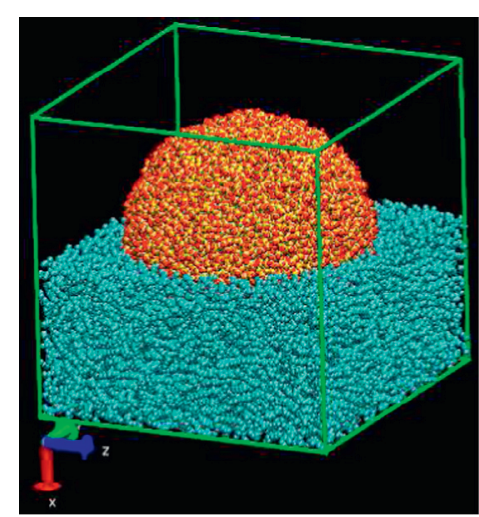

(a)

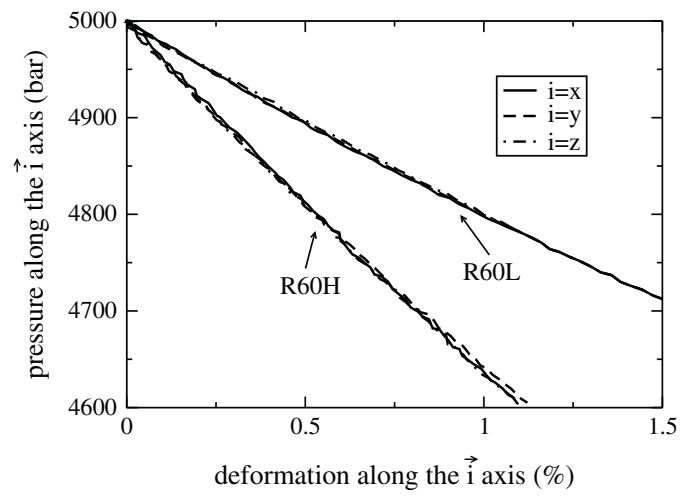

(b)

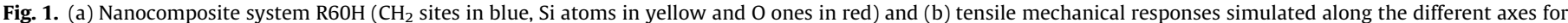

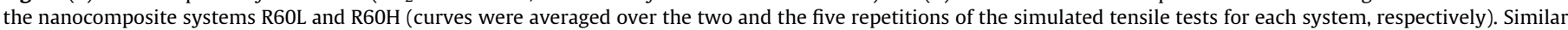

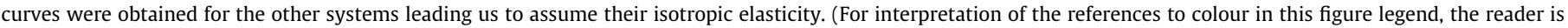
referred to the web version of this article.)

small changes in the box size and shape of a large box and relatively large changes in the size and shape of a small box. It was found that for systems that used the 640-chain polymer matrix a $\tau_{P}$ of 0.1 ps was required to ensure that the box responded fast enough to keep the measured pressure tensor components close to the required ones for the rate of change of $\pm 1 \mathrm{bar}^{\mathrm{ps}}{ }^{-1}$. For the 80 -chain matrix systems, which are about half the size of the 640 -chain systems, a $\tau_{P}$ two times larger of 0.2 ps was, logically, found to be satisfactory. Similarly for systems using the 10-chain matrix a $\tau_{P}$ of $0.4 \mathrm{ps}$ was used. A $\tau_{P}$ of $0.4 \mathrm{ps}$ was also used for the pure silica system.

Tests on specific systems were always started using the same initial positions of atoms as taken from previously relaxed configurations in order to avoid as much as possible differences in relaxational history. Velocity components of each atom were randomised at the start of each simulation with values being taken from a Gaussian distribution coherent with the required temperature. To establish a baseline in each case the first 100 ps were carried out under the standard conditions where the on-diagonal elements of the required pressure tensor were maintained at 5000 bars and the off-diagonal components at zero. After the $100 \mathrm{ps}$ of baseline the non-equilibrium experiments were initiated by ramping up, or down, the relevant components of the required pressure tensor. Results for Young's modulus, $E^{\mathrm{MD}}$, were averaged over experiments performed along the $\vec{x}, \vec{y}$ and $\vec{z}$ axes and for the shear modulus, $\mu^{\mathrm{MD}}$, along the $\{\vec{x}, \vec{y}\},\{\vec{x}, \vec{z}\}$ and $\{\vec{y}, \vec{z}\}$ planes. So for each system seven separate mechanical tests were simulated: one hydrostatic compression, three tensile tests and three shear tests.

For the systems using the 10-chain polymer matrix significant differences were obtained between repeated simulations despite the initial coordinates being the same. To converge the average results, each test was repeated 30 times with a different set of initial random velocities. Thus for the $\mathrm{R} 15 \mathrm{~L}$ and $\mathrm{R} 30 \mathrm{H}$ systems $7 \times 30=210$ separate simulations were carried out in each case. For the 80-chain systems five independent starts was sufficient and for the 640-chain systems the results were averaged over two repeated runs, even though the results in these large systems were virtually indistinguishable. For the pure silica system the simulations were repeated 10 times.

Shear and Young's moduli were determined from the slopes of the corresponding stress-strain curves in the limit of small strains, typically $\approx 1 \%$ in the case of the pure polymers and composites and correspondingly less $(<0.01 \%)$ for the pure silica. The bulk modulus was determined from $k^{\mathrm{MD}}=\rho d P / d \rho$ using the limiting slope of the pressure vs. material density plots, $P=(1 / 3) \operatorname{tr}(\underline{P})$ and $\rho$ respectively, during hydrostatic compressions. Poisson's ratio is defined as the limiting low strain value of the contraction ratio, i.e., the negative ratio of the transversal strain to the elongational strain, in a uniaxial tension test. At low strains, however, the natural fluctuations in the box shape and size lead to large uncertainties in this ratio; so instead we choose to compare values of the contraction ratio at a strain of $1 \%$, in the case of the pure polymer and composite systems. For the pure silica system the contraction ratio was obtained from the average value in the range of strain from $0.04 \%$ to $0.1 \%$. The elastic moduli identified for the various systems are provided and discussed in Section 4.1 (see Table 1).

\subsection{Interphase of disturbed matrix}

In agreement with many other studies in the literature (see Section 1), the characterisation of the molecular structure of the polymer matrix in the various nanocomposite systems simulated has shown the existence of an area of disturbed matrix surrounding the fillers [28]. The behaviour of different quantities such as the mass and conformer distributions, the alignment of the polymer chains or their mobility according to the distance from the nanoparticles were studied. They have shown that, between 0 and about $2 \mathrm{~nm}$ from the particle surface, the polymer chains tend to form layers that are aligned tangentially toward the particle surface and their mobility is diminished. The range of these disturbances was approximately the same for all the nanocomposites simulated, whatever the particle size or volume fraction with respect to the scattering of the results. Thus, in the following, the nanoparticles are assumed to be coated by an interphase of disturbed matrix with a fixed thickness of $2 \mathrm{~nm}$ but with unknown mechanical properties. These coated particles are embedded in the pure matrix. More details regarding the characterisation of the molecular structure of the various systems studied are provided in our previous paper [28].

\section{Micromechanical models and particle size effect}

Taking into account the existence of the aforementioned interphase of disturbed matrix, this section is devoted to the introduction of some outcomes of the MD simulations in micromechanical 
Table 1

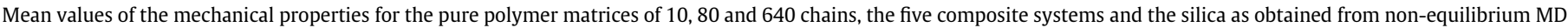

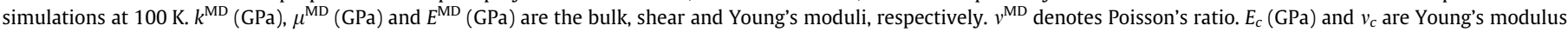

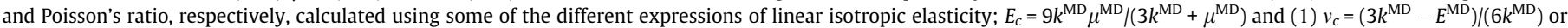
(2) $v_{c}=\left(3 k^{\mathrm{MD}}-2 \mu^{\mathrm{MD}}\right) /\left(2\left(3 k^{\mathrm{MD}}+\mu^{\mathrm{MD}}\right)\right)$. Also given are the estimated volume fractions of filler $f_{1}^{\mathrm{MD}}(\%)$ as obtained previously by a probe insertion technique [28].

\begin{tabular}{|c|c|c|c|c|c|c|c|c|c|}
\hline & 10-chain matrix & 80-chain matrix & 640-chain matrix & $\mathrm{R} 15 \mathrm{~L}$ & R30L & R60L & $\mathrm{R} 30 \mathrm{H}$ & $\mathrm{R} 60 \mathrm{H}$ & Pure silica \\
\hline$k^{\mathrm{MD}}$ & $4.4 \pm 0.2$ & $4.56 \pm 0.09$ & $4.68 \pm 0.04$ & $4.34 \pm 0.09$ & $4.80 \pm 0.06$ & $4.82 \pm 0.09$ & $5.17 \pm 0.12$ & $5.9 \pm 0.2$ & $28 \pm 2$ \\
\hline$\mu^{\mathrm{MD}}$ & $0.617 \pm 0.005$ & $0.647 \pm 0.004$ & $0.651 \pm 0.004$ & $0.664 \pm 0.005$ & $0.702 \pm 0.005$ & $0.712 \pm 0.002$ & $0.925 \pm 0.005$ & $0.995 \pm 0.004$ & $26.3 \pm 0.8$ \\
\hline$E^{\mathrm{MD}}$ & $1.86 \pm 0.02$ & $1.84 \pm 0.01$ & $1.83 \pm 0.01$ & $1.90 \pm 0.02$ & $1.96 \pm 0.01$ & $2.00 \pm 0.01$ & $3.17 \pm 0.02$ & $3.61 \pm 0.03$ & $54 \pm 2$ \\
\hline$v^{\mathrm{MD}}$ & $0.433 \pm 0.006$ & $0.433 \pm 0.003$ & $0.434 \pm 0.002$ & $0.431 \pm 0.006$ & $0.426 \pm 0.007$ & $0.427 \pm 0.002$ & $0.388 \pm 0.004$ & $0.386 \pm 0.004$ & $0.223 \pm 0.007$ \\
\hline$E_{c}$ & $1.77 \pm 0.03$ & $1.85 \pm 0.02$ & $1.87 \pm 0.01$ & $1.90 \pm 0.02$ & $2.00 \pm 0.01$ & $2.04 \pm 0.02$ & $2.62 \pm 0.02$ & $2.83 \pm 0.03$ & $60 \pm 1$ \\
\hline$v_{c}^{(1)}$ & $0.43 \pm 0.02$ & $0.43 \pm 0.01$ & $0.435 \pm 0.004$ & $0.43 \pm 0.01$ & $0.43 \pm 0.01$ & $0.43 \pm 0.01$ & $0.40 \pm 0.01$ & $0.40 \pm 0.01$ & $0.17 \pm 0.01$ \\
\hline$v_{c}^{(2)}$ & $0.43 \pm 0.01$ & $0.432 \pm 0.04$ & $0.434 \pm 0.002$ & $0.427 \pm 0.004$ & $0.430 \pm 0.002$ & $0.430 \pm 0.004$ & $0.42 \pm 0.01$ & $0.42 \pm 0.01$ & $0.14 \pm 0.01$ \\
\hline$f_{1}^{\mathrm{MD}}$ & & & & $3.62 \pm 0.01$ & $4.09 \pm 0.02$ & $4.28 \pm 0.01$ & $25.57 \pm 0.02$ & $26.42 \pm 0.01$ & \\
\hline
\end{tabular}

models in view of the investigation of particle size effects. It shows how an absolute particle size effect can be introduced into micromechanical models as proposed by Marcadon et al. in [23]. To illustrate such a development, only a simple model derived from the classical dilute approximation of the Eshelby's inhomogeneity problem [36] is considered here, but more details are available in the aforementioned paper of Marcadon and co-workers. Note that such a micromechanical analysis could be performed numerically through 'unit-cell' models and the finite element method. Although this would offer the advantage of using the same assumption of periodicity as in MD simulations, it has been preferred to adopt here the framework of random heterogeneous materials which looks more realistic and to have recourse to quasi-analytical treatments in order to obtain an explicit size dependency of the overall behaviour and general properties, instead of the numerical solution of various specific cases.

In the following part of the paper, second-order tensors are still underlined twice whereas fourth-order tensors are indicated with bold characters.

\subsection{Definition of the Representative Volume Element}

The first step of micromechanical approaches is the definition of the Representative Volume Element (RVE) which has to be at once large enough to be statistically representative of the microstructure and to have an overall behaviour representative of the material macroscopic behaviour but small enough to be consistent with further treatments at a larger scale based on continuum mechanics equations. In the case considered here, particulate elastic nanocomposites similar to the MD ones are constituted with spherical monodisperse nanoparticles isotropically (instead of periodically) distributed in a polymer matrix. Each particle is coated with an interphase of disturbed matrix with specific elastic properties. The volume fraction of the 'inclusion phase' $f_{1}$ is fixed and the interphase thickness $t_{\text {int }}$ is independent of the inclusion radius $R_{\text {inc }}$. Let $f_{2}$ and $f_{3}$ denote the volume fractions of the interphase material and of the matrix, respectively, with $f_{1}+f_{2}+f_{3}=1$.

Thus, three different phases $(i)$ are considered, with the moduli $k_{i}, \mu_{i}, E_{i}$ and the Poisson's ratio $v_{i}$. Let $V$ denote the volume of the RVE and $m$ the number of coated inclusions in it (Fig. 2).

Obviously, we have the relations:

$f_{1}=m \frac{4}{3} \pi \frac{R_{\mathrm{inc}}{ }^{3}}{V}$ and $f_{1}+f_{2}=m \frac{4}{3} \pi \frac{\left(t_{\mathrm{int}}+R_{\mathrm{inc}}\right)^{3}}{V}$

Consequently, as already mentioned in [23], the volume fractions of the inclusions and the interphase are linked by:

$f_{2}=f_{1}\left(\left(1+\frac{t_{\text {int }}}{R_{\text {inc }}}\right)^{3}-1\right)$

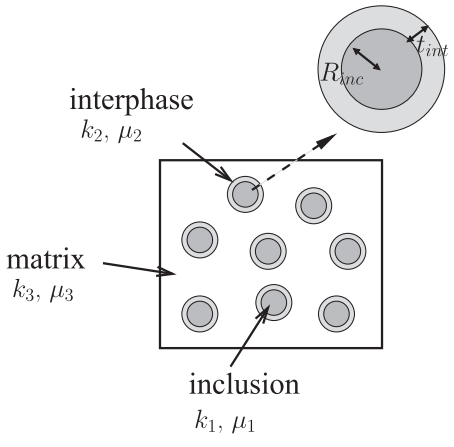

Fig. 2. Definition of the RVE.

As mentioned before, for fixed volume fraction of inclusions and interphase thickness, decreasing the particle size implies an increase of the interphase volume fraction: this is likely to result in an increasing influence of the interphase on the nanocomposite overall mechanical behaviour.

In what follows, we assume a small volume fraction of inclusions and a simple treatment based on a classical 'point approach' is proposed: it refers to the dilute approximation which derives straightforwardly from Eshelby's solution [36] of the ellipsoidal inhomogeneity problem and from the closed form solution of the problem of a coated sphere embedded in an infinite matrix [42]. A more complex treatment, based on the definition of some 'Morphologically Representative Patterns' (MRPs) instead of points [38], could have been proposed but it would have required more information on both the inclusion size dispersion and spatial distribution than the ones available here. The introduction of size effects in the MRP-based approach, and its comparison with the predictions of the classical point approach, have been investigated previously by Marcadon et al. [23].

\subsection{Improved dilute coated inclusion model}

This model, denoted IDCI model in the following, ensues from the classical dilute inclusion-matrix approximation which considers that, when identical inhomogeneities in a sufficiently low volume fraction are dispersed in a matrix, their individual mechanical state can be approached by Eshelby's solution for a single inhomogeneity embedded in an infinite matrix [36,37,39]. Similarly, we assume that, as long as their volume fraction is very low, identical spherical coated inclusions dispersed in an infinite matrix subjected to the strain $\underline{E}$ (or the stress $\underline{\underline{\Sigma}}$ ) at infinity behave identically (see Fig. 3). Nevertheless, a classical 'renormalisation' treatment, which is of current use, e.g., for the Mori-Tanaka model [40] and the self-consistent scheme [41], has been applied in order to extend the validity domain to larger coated inclusion volume fractions. Indeed, due to the fact that reducing the particle radius 


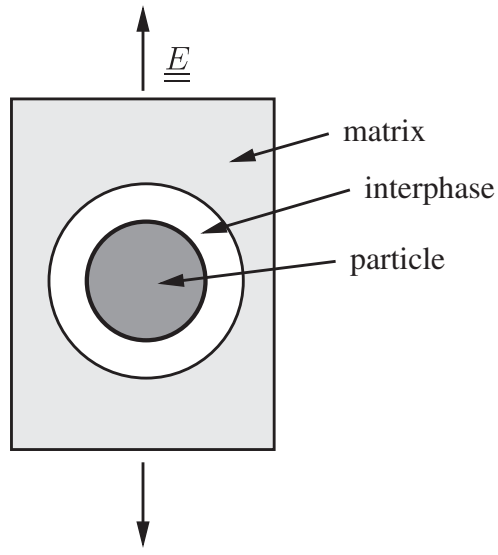

Fig. 3. Assumed situation of individual coated inclusions.

results in an increase of the volume fraction of interphase, hence of coated inclusions, the dilution assumption can be violated for the coated inclusions when $f_{1}$ is very small. This treatment consists in subjecting the system of Fig. 3 to some auxiliary strain tensor $\underline{\underline{E_{0}}}$ (instead of the actual macroscopic strain $\underline{\underline{E}}$ ) at infinity; this strain $\underline{\underline{E_{0}}}$ is then derived from the condition that the resulting average strain in the three-phase material is still equal to $\underline{E}$. More details on this kind of treatment are available in Marcadon et al. [23].

According to [42], for isotropic elasticity, the spherical and deviatoric parts of the average strain tensor in the inclusion $\left(\theta_{1}\right.$ and $\left.\underline{\underline{e}}_{1}\right)$ and the interphase $\left(\theta_{2}\right.$ and $\left.\underline{\underline{e_{2}}}\right)$, with $\underline{\underline{\varepsilon_{i}}}=\left(\theta_{i} / 3\right) \underline{\underline{\delta}}+\underline{\underline{e_{i}}}$, where $\underline{\underline{\delta}}$ denotes the second-order identity tensor, read

$\left\{\begin{array}{l}\theta_{1}=A_{s 1} \theta_{3} \\ \theta_{2}=A_{s 2} \theta_{3} \\ \underline{\underline{e_{1}}}=A_{d 1} \underline{\underline{e_{3}}} \\ \underline{\underline{e_{2}}}=A_{d 2} \underline{\underline{e_{3}}}\end{array}\right.$

as functions of the spherical and deviatoric parts of the average strain tensor in the matrix $\left(\theta_{3}\right.$ and $\left.\underline{e}_{3}\right)$. The expressions of $A_{s 1}, A_{d 1}$, $A_{s 2}$ and $A_{d 2}$, denoting the spherical and deviatoric parts of the strain concentration tensors $\mathbf{A}_{1}$ and $\mathbf{A}_{2}$ for the phases (1) and (2) respectively, are given in Appendix A. They are functions of the characteristic lengths of the nanocomposites through the parameter $c$ which is given by $c=\left(R_{\mathrm{inc}} /\left(t_{\mathrm{int}}+R_{\mathrm{inc}}\right)\right)^{3}$.

The resolution of the problem get finally (see [23] for details) for the elastic moduli $\mathbf{C}^{\text {eff }}$ of the composite:

$\mathbf{C}^{\mathrm{eff}}=\left(f_{1} \mathbf{C}_{1}: \mathbf{A}_{1}+f_{2} \mathbf{C}_{2}: \mathbf{A}_{2}+f_{3} \mathbf{C}_{3}: \mathbf{I}\right):\left(f_{1} \mathbf{A}_{1}+f_{2} \mathbf{A}_{2}+f_{3} \mathbf{I}\right)^{-1}$

Using the decomposition into spherical and deviatoric parts, the effective shear and bulk moduli are given by ([23], Eq. (19)):

$$
\left\{\begin{array}{l}
\mu^{\text {eff }}=\mu_{3}+\frac{f_{1}\left(\mu_{1}-\mu_{3}\right) A_{d 1}+f_{2}\left(\mu_{2}-\mu_{3}\right) A_{d 2}}{f_{3}+f_{1} A_{d 1}+f_{2} A_{d 2}} \\
k^{\text {eff }}=k_{3}+\frac{f_{1}\left(k_{1}-k_{3}\right) A_{s}+f_{2}\left(k_{2}-k_{3}\right) A_{s 2}}{f_{3}+f_{1} A_{s 1}+f_{2} A_{s 2}}
\end{array}\right.
$$

Obviously, the expected size effect is taken into account by the model: for a given inclusion volume fraction $f_{1}$, as soon as the interphase thickness does not depend linearly on the inclusion size (which means that $t_{\text {int }} / R_{\text {inc }}$ is varying when $R_{\text {inc }}$ is modified), the volume fraction $f_{2}$ is varying too and so do the effective moduli $k^{\text {eff }}$ and $\mu^{\text {eff }}$

\subsection{Illustrative examples}

To illustrate the foregoing developments, the predictions of the IDCI model are compared for four sets of variable parameters, as a function of the inclusion radius $R_{\text {inc }}$ : two volume fractions of inclusions and, for each one, two different cases of interphase stiffness, i.e., with an interphase softer or stiffer than the matrix. The fixed parameters are: $\mu_{1}=20 \times \mu_{3}, \quad v_{1}=0.2, \quad v_{2}=0.47, \quad v_{3}=0.43$, $t_{\text {int }}=1 \mathrm{~nm}$. For set $1, f_{1}=5 \%$ and $\mu_{2}=0.5 \times \mu_{3}$. For set $2, f_{1}=5 \%$ and $\mu_{2}=2 \times \mu_{3}$. For set $3, f_{1}=40 \%$ and $\mu_{2}=0.5 \times \mu_{3}$. For set 4 , $f_{1}=40 \%$ and $\mu_{2}=2 \times \mu_{3}$.

Note that, referring to Eqs. (2) and (3), the inclusion radius has been assigned to be larger than a minimum value:

$R_{\min }=\frac{t_{\text {int }}}{\sqrt[3]{f_{\max } / f_{1}}-1}$

where $f_{\max }$ is the expected maximum volume fraction of coated inclusions. As a rough estimate of this maximum fraction, it has been taken here equal to 0.74 , which would correspond to a compact face centred cubic lattice of coated inclusions.

The evolution of the normalised effective shear modulus is plotted in Fig. 4 as a function of the particle radius. This figure clearly shows that the proposed models can actually take a particle size effect into account; this size effect is an over-stiffening one when the interphase is stiffer than the matrix and an under-stiffening one in the reverse case. For an interphase stiffer than the matrix, adding the rigid nanoparticle is expected to have a stiffening effect on the overall behaviour of the nanocomposite that increases with a decreasing particle size. On the contrary, for an interphase softer than the matrix, the expected stiffening effect tends to vanish when decreasing the particle size; indeed an overall softening effect is predicted as a limiting case for small particle sizes and volume fractions. As expected, the stiffening effect is the stronger the larger the volume fraction of inclusions.

Furthermore, it is worth noting that in all cases the particle size effect tends to vanish when the particle radius is large enough, the interphase becoming negligible. Consequently, the effective behaviour of the nanocomposite is predicted to tend toward that of a classical composite made of the inclusions and the matrix only. This asymptotic behaviour depends only on the volume fraction of inclusions and the elastic moduli of the inclusion and matrix phases, and no longer on the inclusion size. These asymptotic values for ratios $\mu^{\text {eff }} / \mu_{3}$ can be computed from the Mori-Tanaka model [40] through the definition of the Hill tensor [43]. They equal about 1.1 for sets 1 and 2 and 2.3 for sets 3 and 4 , respectively.

Note in conclusion that similar tendencies have been obtained for the normalised effective bulk modulus. They have not been reported here for the sake of brevity.

\section{Molecular modelling of size effect on mechanical properties of the nanocomposites}

In this section mechanical properties obtained through MD simulations are summarised and discussed first. Then a confrontation

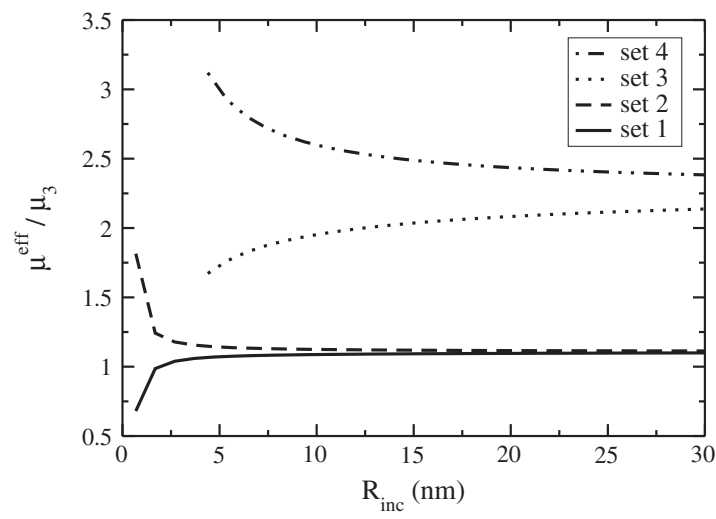

Fig. 4. Prediction of a particle size effect in the IDCI model. 
between MD results and the IDCI model is proposed in order to explain these results and to characterise the elastic moduli of the interphase using an inverse method.

\subsection{Particle size effect observed through molecular modelling}

The different elastic moduli have been determined for the five nanocomposites, the three pure polymer matrices and the pure silica by performing appropriate mechanical tests using MD, as described in Section 2.

In Table 1 only mean values for the different moduli are given along with the estimates of the volume fractions of filler as obtained previously using a probe insertion method [28]. First, it can be noticed that, if the mean values for the different elastic moduli are considered for each nanocomposite, the relations of linear isotropic elasticity hold reasonably well for systems with a low volume fraction of inclusions. The same conclusion can be drawn for pure polymer matrices. For silica and systems with a high volume fraction of inclusions, these relations are less well verified, probably because of the lattice construction of the silica model (see Section 2), but the difference remains quite low. Thus, the different materials simulated can be considered as isotropic linear elastic materials.

Now if we compare the values of the moduli of the different nanocomposites having a low volume fraction of inclusions to each other and to their corresponding pure matrices, we can see that, where a difference is significant with respect to the errors, there is a mechanical reinforcement due to the presence of the inclusions. Except for the system R15L, the bulk, shear and Young's moduli of the composites are higher than those of the pure matrix. Because of the low volume fraction of inclusions considered here, adding the nanoparticles has no influence on the Poisson's ratio of the composite relative to the pure matrix. For the bulk, shear and Young's moduli a particle size effect is also seen; increasing the particle radius induces an increase of the moduli, although care has to be taken here as there is a decrease in volume fraction as filler particles decrease in size too (about 15\%).

The particle size under-stiffening effect is also evident for the nanocomposites with a high volume fraction of inclusions; for those systems, the decrease in filler volume fraction is only about $3 \%$. In this case, it is worth noting that Poisson's ratio substantially decreases when adding the rigid particle. As expected, the comparison between the nanocomposites having the same particle radius shows that the higher the volume fraction of inclusions, the stiffer the composite for a given particle size.

If the particular case of the nanocomposite R15L is addressed, it is worth noting that its bulk modulus is slightly lower than that of the corresponding matrix despite the presence of the rigid silica nanoparticle. Although the difference is less than the associated error, such a limit consequence of the under-stiffening phenomenon has already been seen in other MD simulations [16,17]. In order to explain it a confrontation between MD simulations and micromechanical approaches to determine the mechanical properties of the interphase is developed.

\subsection{Determination of the interphase mechanical properties}

\subsubsection{Method for the confrontation between MD and micromechanical approaches}

Assuming the existence of an interphase of known constant thickness, of a value obtainable from MD simulations, mechanical properties of the interphase are determined fitting the moduli obtained by MD thanks to the IDCI model. The methodology is detailed below in the case of the low volume fraction of inclusions.

Properties obtained by molecular modelling are:
- the volume fraction of inclusions $f_{1}=4.5 \%$ (target value for systems with a low volume fraction of inclusions);

- the elastic moduli of the inclusion and the matrix phases: $k_{1}=28 \mathrm{GPa}, \mu_{1}=26,3 \mathrm{GPa}$ and $v_{1}=0.22$ for the inclusion and $k_{3}=4.68 \mathrm{GPa}, \mu_{3}=0.651 \mathrm{GPa}$ and $v_{3}=0.434$ for the matrix (values for the 640-chain matrix), respectively. Since MD simulations give access to Poisson's ratios for the inclusion and the matrix, values of $v_{1}$ and $v_{3}$ are explicitly introduced in the equations, contrarily to $v_{2}$ which is calculated from $k_{2}$ and $\mu_{2}$ using relations of the linear isotropic elasticity;

- the effective elastic moduli of the simulated nanocomposites $\left(k^{\mathrm{MD}}\right.$ and $\left.\mu^{\mathrm{MD}}\right)$ for three different particle sizes $\left(R_{\mathrm{inc}}=1.5,3\right.$ and $\left.6 \mathrm{~nm}\right)$;

- the interphase thickness $t_{\text {int }}$ that equals $2 \mathrm{~nm}$ (see [28]).

As shown in Table 1 and in [28], the atomistic representation of the nanocomposites through MD results in a variation of both the mechanical (elastic moduli) and morphological (interphase thickness, inclusion size and volume fraction) properties identified for the different constitutive phases according to the size of the systems and the roughness of the interfaces. Such atomistic pieces of information cannot be accounted for in micromechanical approaches used here, deriving from continuum mechanics, and in which interfaces are assumed to be perfect. Even if it is beyond the scope of the present work, the transition from continuum to atomistic approaches should be more thoroughly investigated with the purpose to improve the dialogue between these different scales. Thus, to address these limitations of the comparison between MD and micromechanics, we have assumed that elastic moduli of the matrix do not depend on the size of the system and equal the ones of the 640-chain matrix. Similarly, target values have been considered for the inclusion radii and the volume fractions.

To simplify the problem of the confrontation between MD and micromechanical approaches, interphase behaviour is assumed to be independent of the particle size, isotropic and uniform. If we look at the evolution of the polymer density and the local orientation of polymer chains as functions of the distance to the particle surface $[16,28]$, the properties certainly vary gradually and as a consequence the mechanical behaviour of the interphase may be radial orthotropic. Nevertheless, to simplify the confrontation and because of the lack of local information on the behaviour of the interphase provided by $\mathrm{MD}$, the interphase behaviour is assumed to remain isotropic linear elastic like the inclusion and matrix phases.

The choice of the IDCI model is justified by the fact that, agreeing with Section 3.3, the volume fraction of coated inclusions increases with a decreasing particle size. Thus for small particles, the assumption of dilution is no longer relevant for coated particles. By using the micromechanical model, elastic moduli of the interphase are determined by an inverse method in order to understand the influence of its mechanical behaviour on the overall one of the nanocomposites. For the confrontation the bulk and shear moduli are preferred in accordance to the micromechanical approach. At the temperature of $100 \mathrm{~K}$, molecular simulations have revealed that mechanical behaviours of the different studied systems (the pure silica, the pure matrix and the different nanocomposites) can be considered as linear isotropic elastic. That agrees with assumptions of the micromechanical models described here above.

The problem of the confrontation between periodic and nonperiodic systems arises with the confrontation between molecular simulations and micromechanical approaches. The dispersion of the particles inside the nanocomposites realised by MD is periodic, and yet on the contrary self-consistent micromechanical models 
are generally used to deal with composites with randomly dispersed particles. However, a previous confrontation between the three-phase model and a periodic homogenisation model, taking both into account the 'inclusion-matrix' morphology of fibre composites, yields very close results even though the periodic and the three-phase models correspond, respectively, to well-ordered and to disordered configurations [44]. According to these results, the different arrangements corresponding to these two models (wellordered in the case of the MD simulations and disordered in the case of the IDCI model) are supposed to have no significant effect on the confrontation.

\subsubsection{Characterisation of the interphase}

Given the equations of the IDCI model (Eq. (6)) it is possible to determine the elastic behaviour of the interphase (here $k_{2}$ and $\mu_{2}$ ) from the knowledge of the behaviour of the inclusions, the matrix and the composite material. By substituting the expressions for $A_{s 1}$ and $A_{s 2}$ (Eq. (9)) in Eq. (6), $k_{2}$ is then given by:

$k_{2}=\frac{f_{3} \beta\left(k_{3}-k^{\mathrm{eff}}\right)+4 \mu_{2} f_{1}\left(3 k_{3}+4 \mu_{3}\right)\left(k_{1}-k^{\mathrm{eff}}\right)-f_{2} k^{\mathrm{eff}}\left(3 k_{3}+4 \mu_{3}\right)\left(3 k_{1}+4 \mu_{2}\right)}{f_{3} \gamma\left(k^{\mathrm{eff}}-k_{3}\right)+3 f_{1}\left(3 k_{3}+4 \mu_{3}\right)\left(k^{\text {eff }}-k_{1}\right)-f_{2}\left(3 k_{3}+4 \mu_{3}\right)\left(3 k_{1}+4 \mu_{2}\right)}$

with $\gamma=3\left(3 k_{1}+4 c \mu_{3}\right)+12 \mu_{2}(1-c) \quad$ and $\quad \beta=12 \mu_{3} k_{1}(1-c)$ $+4 \mu_{2}\left(4 \mu_{3}+3 c k_{1}\right)$.

If $k^{\mathrm{MD}}$ and $\mu^{\mathrm{MD}}$ denote respectively the bulk modulus and the shear modulus of the composite material provided by MD, the bulk modulus of the interphase is then determined from Eq. (8) where $k^{\mathrm{MD}}$ is substituted for $k^{\text {eff. }}$. By using a classical dichotomy method the shear modulus of the interphase is the value leading to a vanishing value of the expression $\left(\mu^{\mathrm{MD}}-\mu^{\mathrm{eff}}\right)$ where $\mu^{\text {eff }}$ is given by Eq. (6).

Due to the uncertainties of molecular simulation results, there is no chance to find the same couple $\left(k_{2}, \mu_{2}\right)$ for the three different particle sizes. As a consequence, taking into account errors on the elastic moduli provided by MD, the domains of couples $\left(k_{2}, \mu_{2}\right)$ solutions of the problem $k^{\text {eff }}\left(k_{2}, \mu_{2}\right)=k^{\mathrm{MD}} \pm 5 \%$ and $\mu^{\text {eff }}\left(k_{2}\right.$, $\left.\mu_{2}\right)=\mu^{\mathrm{MD}} \pm 5 \%$ for each particle radius are determined and the elastic moduli of the interphase are defined as the isobarycenter of the intersection (see Fig 5(a)).

As a matter of fact, as illustrated in Section 3.3 the size effect deduced from the micromechanical models is significant only in a small range of particle radius, around the interphase thickness. Models show that rapidly the role of the interphase becomes negligible and the mechanical behaviour of the composite reaches an asymptotic value which depends only on mechanical properties of both the inclusion and the matrix, and on the inclusion volume fraction. Thus, increasing the particle radius considerably widens the number of couples $\left(k_{2}, \mu_{2}\right)$ solutions and in practice the intersection of the different domains is very close to the one obtained for the smallest particle radius.

As a consequence $k_{2}$ and $\mu_{2}$ equal respectively $3.80 \mathrm{GPa}$ and $0.580 \mathrm{GPa}$. For comparison, these moduli respectively equal $4.68 \mathrm{GPa}$ and $0.651 \mathrm{GPa}$ for the pure matrix. Thus the interphase is effectively softer than the pure matrix. Now, if the determined values of $k_{2}$ and $\mu_{2}$ are injected in the IDCI model and if the predicted effective moduli are compared with the one obtained through MD simulations (Fig. 5(b)), there is a pretty good agreement. Qualitatively, the particle size effect predicted by the micromechanical model is the same as the one observed on the virtual nanocomposites, i.e., an under-stiffening effect. Quantitatively, the differences between micromechanical predictions and MD values remain low. Similarly to illustrative examples of Section 3.3, the asymptotic values for $\mu^{\text {eff }} / \mu_{3}$ and $k^{\text {eff }} / k_{3}$ have been computed and they equal approximately 1.10 and 1.04 , respectively.

\subsubsection{Under-stiffening effect}

Fig. 5(b) reveals that no stiffening effect exists for the system $\mathrm{R} 15 \mathrm{~L}$ despite the presence of rigid nanoparticles. Actually a critical particle radius exists for which, if the particle size is too small, a softened overall behaviour of the nanocomposite relative to the one of the pure matrix is observed despite the addition of rigid nanoparticles. According to the particle size effects predicted by the micromechanical model (see Section 3.3), this under-stiffening phenomenon could be explained by the fact that, for fixed interphase thickness and volume fraction of inclusions, a decrease of the particle size induces an increase of the volume fraction of interphase softer than the pure matrix. Consequently, if the interphase becomes dominating, the effective behaviour of the nanocomposite can be softer than the matrix one. Such a softened overall behaviour is a limit case which could be observed only for very low volume fractions of inclusions and very small particle sizes.

These results have been asserted by applying the fitting procedure for a different value of $f_{1}$ for systems with a low volume fraction of inclusions. Indeed, the determination of the volume fractions of inclusions for the different nanocomposites, using a random probe insertion technique [28], has revealed that the real volume fractions of inclusions can be significantly lower than the target value because of the atomistic modelling of the particles and their roughness. Whereas the target value equals $4.5 \%$ for the nanocomposites, the real values are about $3.6 \%, 4.1 \%$ and 4.3\% for systems R15L, R30L and R60L, respectively. Thus the fitting procedure has been performed for a mean value of $f_{1}=4.0 \%$. For the sake of brevity corresponding domains and their intersection are not plotted here but the identified interphase moduli are very close to the ones obtained for $f_{1}=4.5 \%$ (for $f_{1}=4.0 \%, k_{2}=3.75 \mathrm{GPa}$ and $\mu_{2}=0.580 \mathrm{GPa}$ ). The identification method proposed appears to be rather insensitive to small variations of the volume fraction of inclusions and, even for a value of $f_{1}=4.0 \%$, a good agreement between MD and micromechanical approaches is still observed (see Fig. 5(b)).

Furthermore, assuming that introducing a volume fraction of inclusions which would depend on their size into the micromechanical model would have a sense, the decrease of the volume fraction of inclusions with their size would result in a decrease of the interphase volume fraction too, for a given interphase thickness. Hence the loss of particle stiffening effect would be balanced by the loss of interphase softening one. This leads us to suppose that the observed overall under-stiffening effect is not an artefact of the comparison between the MD and micromechanical approaches.

\subsubsection{High volume fraction of inclusions: validation of the methodology}

In order to discuss the aforementioned methodology proposed for the inverse identification of the interphase elastic moduli, systems with a high volume fraction of inclusions are considered now. Bulk and shear moduli of the interphase have been determined from the simulations conducted on systems $\mathrm{R} 30 \mathrm{H}$ and $\mathrm{R} 60 \mathrm{H}$, for both the target value of $f_{1}=27.0 \%$ and the mean value of $f_{1}=26.0 \%$ (the volume fraction of inclusions for the system $\mathrm{R} 30 \mathrm{H}$ equals $\approx 25.6 \%$, whereas it equals $\approx 26.4 \%$ for the system $\mathrm{R} 60 \mathrm{H}$ ). However, for these systems, the problem $k^{\text {eff }}\left(k_{2}, \mu_{2}\right)=k^{\mathrm{MD}} \pm 10 \%$ and $\mu^{\text {eff }}\left(k_{2}, \mu_{2}\right)=\mu^{\mathrm{MD}} \pm 10 \%$ has been solved instead of the problem $k^{\mathrm{eff}}\left(k_{2}, \mu_{2}\right)=k^{\mathrm{MD}} \pm 5 \%$ and $\mu^{\text {eff }}\left(k_{2}, \mu_{2}\right)=\mu^{\mathrm{MD}} \pm 5 \%$ because there was no solution for this initial problem. Indeed, only one value of $\mu_{2}=0.55 \mathrm{GPa}$ is a solution of the first problem for the system $\mathrm{R} 30 \mathrm{H}$, this value being very close to but not included in the interval of solutions $\mu_{2} \in[0.45 ; 0.50]$ for the system R60H. Similar conclusions as those obtained on systems with a low volume fraction of inclusions can be drawn and corresponding fitted moduli are $k_{2}=4.0 \mathrm{GPa}$ and $\mu_{2}=0.50 \mathrm{GPa}$ for $f_{1}=27.0 \%$ (see Fig. $6(\mathrm{a}$ ) and 


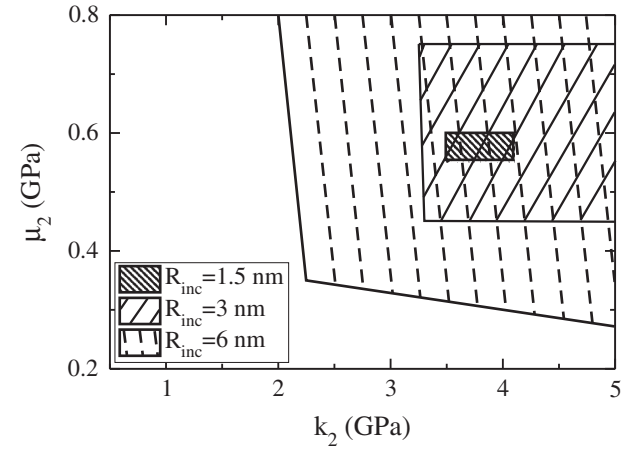

(a)

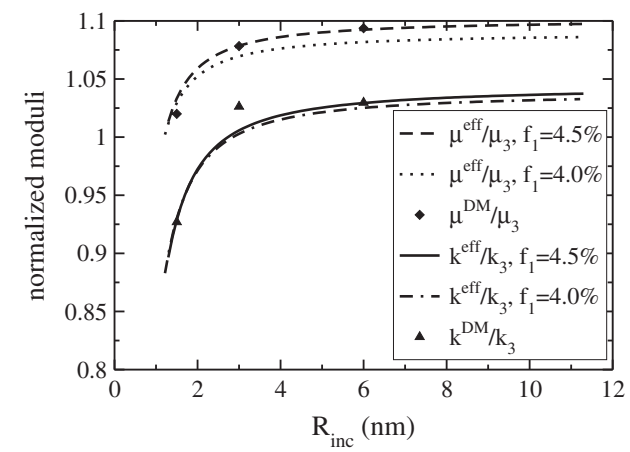

(b)

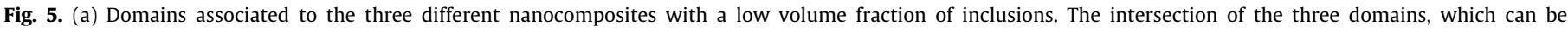

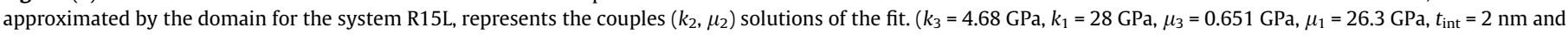
$f_{1}=4.5 \%$ ) and (b) confrontation between MD simulations and the IDCI model for systems having a low volume fraction of inclusions.

(b)), but also for $f_{1}=26.0 \%$. For systems with a high volume fraction of inclusions, the asymptotic values for $\mu^{\text {eff }} / \mu_{3}$ and $k^{\text {eff }} / k_{3}$, i.e. when neglecting the interphase, equal about 1.79 and 1.33 , respectively.

These results have to be considered carefully since for $f_{1}=27.0 \%$ the critical radius $R_{\min }$ equals $5 \mathrm{~nm}$, hence the system $\mathrm{R} 30 \mathrm{H}$ no longer obeys all the assumptions of the micromechanical approach and this particular system represents a nanocomposite in which there is only the disturbed matrix between two neighbour inclusions. It could explain the difference between the values of $k_{2}$ and $\mu_{2}$ identified from systems with a low volume fraction of inclusions and those identified from systems with a high volume fraction of inclusions. Nevertheless, these values are quite close to the ones obtained considering systems having a low volume fraction of inclusions. They assert the fact that the elastic moduli of the interphase of disturbed matrix are lower than those of the pure matrix, of about $15 \%$ for both the bulk and shear moduli, that induces the under-stiffening effect observed through MD simulations.

With the purpose to verify the relevance of the IDCI model for such a high volume fraction of inclusions, the identification procedure described above has been applied replacing the IDCI model by the ' $3+1$-phase' model developped by Hervé and Zaoui [45] (see Eqs. (46) and (51) of [45] for the expressions of $k^{\text {eff }}$ and $\mu^{\text {eff }}$ in that case). In their previous work, Marcadon and co-workers [23] have shown that the renormalisation treatment proposed in the IDCI model is no longer sufficient when increasing the volume fraction of inclusions and, in that case, the $3+1$-phase model gives more accurate estimates. However, because of a rather low contrast between the elastic moduli of the matrix and those of the inclusion and the interphase especially, even for a volume fraction of inclusions of about $26-27 \%$ there is a very good agreement between the values of $k_{2}$ and $\mu_{2}$ identified thanks to the IDCI model and those identified by using the $3+1$-phase model (see Table 2 ). The IDCI model is thus relevant for the present work.

The fact that the interphase is softer than the pure matrix despite a lower mobility of the polymer chains in this disturbed area (see [28]) seems to be contradictory since a more brittle and stiffer polymer matrix would be expected by decreasing chains mobility. Nevertheless, it can be noticed that here the disturbed matrix interphase is a confined matter which probably has a strongly anisotropic behaviour due to the rearrangement of the polymer chains, so that has nothing in common with a bulk polymer. One possible scenario that could explain such contradictory observations might ensue from the reorientation of the polymer chains in concentric layers of different densities in the interphase with a lower cohesion between themselves and a lower entanglement of the polymer chains too. Due to the layers having a lower density than the pure matrix, hence lower mechanical properties, the effective radial and tangential stiffnesses of the interphase should be lower than the bulk ones, with no longer correlation with the intra-layer mobility of the chains. However, this last conclusion remains questionable.

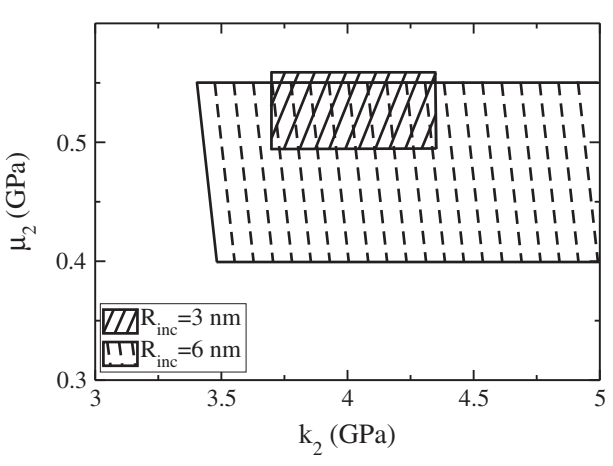

(a)

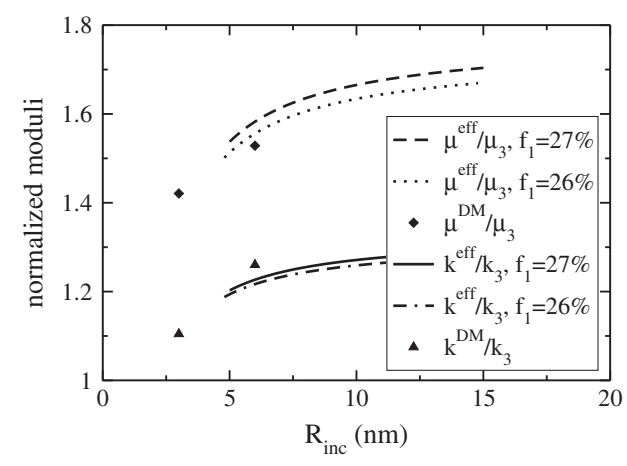

(b)

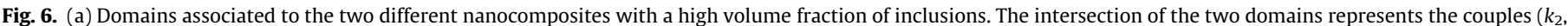

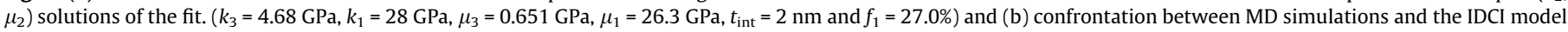
for systems having a high volume fraction of inclusions. 
Table 2

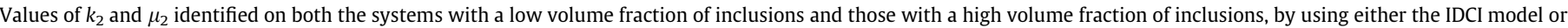
the $3+1$-phase one.

\begin{tabular}{|c|c|c|c|c|c|c|c|c|}
\hline & \multicolumn{2}{|c|}{$f_{1}=4.5 \%$} & \multicolumn{2}{|c|}{$f_{1}=4.0 \%$} & \multicolumn{2}{|c|}{$f_{1}=27.0 \%$} & \multicolumn{2}{|c|}{$f_{1}=26.0 \%$} \\
\hline & IDCI & $3+1$-phase & IDCI & $3+1$-phase & IDCI & $3+1$-phase & IDCI & $3+1$-phase \\
\hline$k_{2}(\mathrm{GPa})$ & 3.80 & 3.80 & 3.75 & 3.75 & 4.0 & 4.03 & 4.0 & 4.03 \\
\hline$\mu_{2}(\mathrm{GPa})$ & 0.580 & 0.575 & 0.580 & 0.575 & 0.50 & 0.525 & 0.50 & 0.525 \\
\hline
\end{tabular}

In the literature, different methodologies have been proposed to have access to the local mechanical properties of confined polymers, see for instance the works of Papakonstantopoulos et al. [46] and Riccardi et al. [47] through MD or the experiments of O'Connell and McKenna [48]. Nevertheless, from a continuum mechanics point of view they show some limitations. Important contributions of side effects are highlighted (surface tension for instance [48]). Such considerations should be negligible when identifying bulk properties such as Young's modulus or Poisson's ratio. Moreover, Riccardi et al. [47] have measured local values for Poisson's ratio higher than 0.5, which is impossible when assuming an isotropic behaviour. Whereas the classical quantities of continuum mechanics identified on periodic systems, even at the atomic scale, are reliable since they are representative of a bulk, in the case of confined matter some care has to be taken when going from the atomic scale to the continuum one. It is an open and a critical issue that needs to be further investigated.

\section{Conclusions}

Simulating virtual nanocomposites using Molecular Dynamics allowed us to analyse a particle size effect on their overall mechanical behaviour. By assuming the existence of an interphase of disturbed matrix surrounding the nanoparticles, with a fixed thickness, a particle size effect can be expressed by micromechanical models. In this paper the micromechanical modelling makes use of a dilute model based on the problem of Eshelby's inhomogeneity. Particularly, an improvement of the dilute coated inclusions model has been proposed to face the problem of the too restrictive dilution assumption for coated inclusions.

The study of the mechanical behaviour of virtual nanocomposites, using Molecular Dynamics simulations, has shown a reinforcement effect on the composite effective moduli and a particle size effect; elastic moduli increase with the particle radius at fixed particle volume fraction. It has been shown, for the particular case of nanocomposites having both a low volume fraction of inclusions and a small particle size, that the effective behaviour of the nanocomposite can even be softer than the one of the pure matrix despite the addition of rigid fillers. The confrontation with the micromechanical modelling and the determination of the elastic moduli of the interphase by an inverse method allowed the under-stiffening particle size effect observed by MD to be explained in the case of an interphase, of fixed thickness, softer than the matrix. In the reverse case, i.e., an interphase stiffer than the matrix, an over-stiffening of the nanocomposite behaviour is predicted by the micromechanical model with a decreasing particle size.

Despite some limitations resulting from the specificities of atomistic and continuum approaches, the interest of the proposed method lies in the fact that a dialogue between distinct physics and material scales has been initiated.

\section{Appendix A}

Expressions of the spherical and deviatoric parts of the strain concentration tensors for the inclusion and interphase, $A_{s 1}, A_{s 2}$, $A_{d 1}$ and $A_{d 2}$ :

$$
\left\{\begin{aligned}
A_{s 1} & =\frac{\left(3 k_{3}+4 \mu_{3}\right)\left(3 k_{2}+4 \mu_{2}\right)}{\left(3 k_{2}+4 \mu_{3}\right)\left(3 k_{1}+4 \mu_{2}\right)+12 c\left(\mu_{3}-\mu_{2}\right)\left(k_{2}-k_{1}\right)} \\
A_{s 2} & =\frac{\left(3 k_{3}+4 \mu_{3}\right)\left(3 k_{1}+4 \mu_{2}\right)}{\left(3 k_{2}+4 \mu_{3}\right)\left(3 k_{1}+4 \mu_{2}\right)+12 c\left(\mu_{3}-\mu_{2}\right)\left(k_{2}-k_{1}\right)} \\
A_{d 1} & =225\left(1-v_{3}\right)\left(1-v_{2}\right) X_{0} \times \frac{-4\left(X_{0}-1\right)\left[\eta_{1} c^{7 / 3}-\eta_{2}\left(7-10 v_{2}\right)\right]+35\left(1-v_{2}\right) \eta_{2}}{4} \\
A_{d 2} & =\frac{15\left(1-v_{3}\right) X_{0}}{1-c} \times \cdots \\
& \ldots \frac{\left(X_{0}-1\right)\left\{A+60 c\left(1-v_{2}\right)\left[\eta_{1} c^{7 / 3}-\eta_{2}\left(7-10 v_{2}\right)\right]\right\}+35\left(1-v_{2}\right) \eta_{2} \eta_{3}(1-c)}{4}
\end{aligned}\right.
$$

with

$$
\left\{\begin{array}{l}
X_{0}=\frac{\mu_{3}}{\mu_{2}} \\
\alpha=\frac{\mu_{1}}{\mu_{2}}-1 \\
\eta_{1}=\left(49-50 v_{1} v_{2}\right) \alpha+35(1+\alpha)\left(v_{1}-2 v_{2}\right)+35\left(2 v_{1}-v_{2}\right) \\
\eta_{2}=\left(7+5 v_{1}\right)(1+\alpha)+4\left(7-10 v_{1}\right) \\
\eta_{3}=2(1+\alpha)\left(4-5 v_{2}\right)+7-5 v_{2} \\
A=-4\left[\eta_{3}-2 \alpha\left(4-5 v_{2}\right) c\right]\left[\eta_{1} c^{7 / 3}-\eta_{2}\left(7-10 v_{2}\right)\right]-126 \alpha \eta_{2} c\left(1-c^{2 / 3}\right)^{2} \\
C=-\left[\eta_{3}+\alpha\left(7-5 v_{2}\right) c\right]\left[4 \eta_{1} c^{7 / 3}+\eta_{2}\left(7+5 v_{2}\right)\right]-126 \alpha \eta_{2} c\left(1-c^{2 / 3}\right)^{2} \\
\Delta=\left[2\left(4-5 v_{3}\right) C+\left(7-5 v_{3}\right) A X_{0}\right]\left(X_{0}-1\right)+\cdots \\
\quad \cdots 525 \eta_{2}\left(1-v_{2}\right)\left[2 \alpha\left(v_{2}-v_{3}\right) c+\left(1-v_{3}\right) \eta_{3}\right] X_{0} .
\end{array}\right.
$$

In these expressions $c=\left(R_{\text {inc }} /\left(t_{\text {int }}+R_{\text {inc }}\right)\right)^{3}$.

\section{References}

[1] J. Móczó, B. Pukánsky, J. Indust. Eng. Chem. 14 (2008) 535-563.

[2] S.Y. Fu, X.Q. Feng, B. Lauke, Y.W. Mai, Composites: Part B 39 (2008) 933-961.

[3] B. Natarajan, Y. Li, H. Deng, L.C. Brinson, L.S. Schadler, Macromolecules 46 (2013) 2833-2841.

[4] Q. Chen, I. Chasiotis, C. Chen, A. Roy, Compos. Sci. Technol. 68 (2008) 31373144.

[5] J. Cho, M.S. Joshi, C.T. Sun, Compos. Sci. Technol. 66 (2006) 1941-1952.

[6] A. Kongsinlark, G.L. Rempel, P. Prasassarakich, Chem. Eng. J. 193-194 (2012) 215-226.

[7] A.C. Steenbrink, V.M. Litvinov, R.J. Gaymans, Polymer 39 (1998) 4817-4825.

[8] N.D. Albérola, K. Benzarti, C. Bas, Y. Bomal, Polym. Compos. 22 (2001) 312-325

[9] A. Lazzeri, S.M. Zebarjad, M. Pracella, K. Cavalier, R. Rosa, Polymer 46 (2005) 827-844.

[10] J. Berriot, F. Lequeux, L. Monnerie, H. Montes, D. Long, P. Sotta, J. Non-Cryst Solids 307-310 (2002) 719-724.

[11] J. Berriot, F. Martin, H. Montes, L. Monnerie, P. Sotta, Polymer 44 (2003) 14371447.

[12] F.W. Starr, T.B. Schroder, S.C. Glotzer, Phys. Rev. E: Statist. Phys. Plasmas Fluids 64 (2001). 021802/1-5.

[13] F.W. Starr, T.B. Schroder, S.C. Glotzer, Macromolecules 35 (2002) 4481-4492

[14] F.W. Starr, J.F. Douglas, S.C. Glotzer, J. Chem. Phys. 119 (2003) 1777-1788.

[15] D. Barbier, D. Brown, A.C. Grillet, S. Neyertz, Macromolecules 37 (2004) 46954710.

[16] D. Brown, P. Mélé, S. Marceau, N.D. Albérola, Macromolecules 36 (2003) 1395 1406.

[17] G.M. Odegard, T.C. Clancy, T.S. Gates, Polymer 46 (2005) 553-562.

[18] A. Ghanbari, T.V.M. Ndoro, F. Leroy, M. Rahimi, M.C. Böhm, F. Müller-Plathe, Macromolecules 45 (2012) 572-584.

[19] T.V.M. Ndoro, M.C. Böhm, F. Müller-Plathe, Macromolecules 45 (2012) 171 179

[20] M. Rahimi, I. Iriarte-Carretero, A. Ghanbari, M.C. Böhm, F. Müller-Plathe, Nanotechnology 23 (2012) 305702.

[21] A. Adnan, C.T. Sun, H. Mahfuz, Compos. Sci. Technol. 67 (2007) 348-356.

[22] J. Cho, C.T. Sun, Comput. Mater. Sci. 41 (2007) 54-62.

[23] V. Marcadon, E. Hervé, A. Zaoui, Int. J. Solids Struct. 44 (2007) 8213-8228.

[24] S. Boutaleb, F. Zaïri, A. Mesbah, M. Naït-Abdelaziz, J.-M. Gloaguen, T. Boukharouba, J.-M. Lefebvre, Int. J. Solids Struct. 46 (2009) 1716-1726.

[25] S. Boutaleb, F. Zaïri, A. Mesbah, M. Naït-Abdelaziz, J.-M. Gloaguen, T. Boukharouba, J.-M. Lefebvre, Procedia Eng. 1 (2009) 217-220. 
[26] S. Saber-Samandari, A. Afaghi-Khatibi, Polym. Compos. 28 (2007) 405-411.

[27] R.D. Peng, H.W. Zhou, H.W. Wang, L. Mishnaevsky Jr., Comput. Mater. Sci. 60 (2012) 19-31.

[28] D. Brown, V. Marcadon, P. Mélé, N.D. Albérola, Macromolecules 41 (2008) 1499-1511.

[29] B.W.H. van Beest, G.J. Kramer, R.A. van Santen, Phys. Rev. Lett. 64 (1990) 1955 1958

[30] K. Vollmayr, W. Kob, K. Binder, Phys. Rev. B 54 (1996) 15808-15827.

[31] D. Brown, J.H.R. Clarke, Macromolecules 24 (1991) 2075-2082.

[32] D. Brown, J.H.R. Clarke, M. Okuda, T. Yamazaki, J. Chem. Phys. 100 (1994) 1684-1692.

[33] D. Brown, J.H.R. Clarke, M. Okuda, T. Yamazaki, J. Chem. Phys. 100 (1994) 6011-6018.

[34] D. Brown, J.H.R. Clarke, M. Okuda, T. Yamazaki, J. Chem. Phys. 104 (1996) 2078-2082.

[35] H.J.C. Berendsen, J.P.M. Postma, W.F. van Gunsteren, A. Di Nola, J.R. Haak, J. Chem. Phys. 81 (1984) 3684-3690.

[36] J.D. Eshelby, Proc. Roy. Soc. London A421 (1957) 376-396
[37] J.D. Eshelby, Prog. Solid State Phys. 3 (1956) 79-144.

[38] M. Bornert, C. Stolz, A. Zaoui, J. Mech. Phys. Solids 44 (1996) 307-331.

[39] A. Einstein, Ann. Phys. 19 (1906) 289-306.

[40] T. Mori, K. Tanaka, Acta Metall. 21 (1973) 571-574.

[41] E. Kröner, J. Phys. F8 (1978) 2261-2267.

[42] E. Hervé, A. Zaoui, Eur. J. Mech. A/Solids 9 (1990) 505-515.

[43] R. Hill, J. Mech. Phys. Solids 13 (1965) 89-101.

[44] E. Hervé, A. Zaoui, Morphological n-layered cylindrical pattern-based micromechenical modeling, in: D.F. Parker, A.H. England (Eds.), IUTAM Symposium on Anisotropy, Inhomogeneity and Nonlinearity in Solid Mechanics, Kluwer Academic Publishers, 1995, pp. 403-408.

[45] E. Hervé, A. Zaoui, Int. J. Eng. Sci. 31 (1993) 1-10.

[46] G.J. Papakonstantopoulos, M. Doxastakis, P.F. Nealey, J.-L. Barrat, J.J. de Pablo, Phys. Rev. E 75 (2007) 031803.

[47] E. Riccardi, M.C. Böhm, F. Müller-Plathe, Phys. Rev. E 86 (2012) 036704

[48] P.A. O'Connell, G.B. McKenna, J. Polym. Sci.: Part B: Polym. Phys. 47 (2009) 2441-2448. 